\title{
Simulation of Magnetization Switching in Nanoparticle Systems
}

\author{
D. Hinzke and U. Nowak \\ Theoretische Physik, Gerhard-Mercator-Universität \\ 47048 Duisburg, Germany
}

Pacs-numbers: 75.10.Hk; 75.40.Mg; 75.40.Gb

\begin{abstract}
Magnetization reversal in magnetic nanostructures is investigated numerically over time-scales ranging from fast switching processes on a picosecond scale to thermally activated reversal on a microsecond time-scale. A simulation of the stochastic Landau-Lifshitz equation of motion is used as well as a time quantified Monte Carlo method for the simulation of classical spin systems modeling magnetic Co nanoparticles. For field pulses larger than the Stoner-Wohlfarth limit spin precession effects govern the reversal behavior of the particle while for lower fields a magnetization reversal is only possible when it is assisted by thermal fluctuations.
\end{abstract}




\section{Introduction}

The miniaturization of magnetic structures plays an important role for fundamental research as well as for technical applications. This leads to an incremental interest in the understanding of the behavior of small magnetic particles and structures down to the nanometer scale. But with decreasing size of the magnetic system thermal activation becomes relevant. Hence, much effort is focused now on the understanding of magnetization dynamics at finite temperatures. [1].

In the following, we briefly describe numerical techniques for the study of magnetization dynamics in nanostructures, modeled as classical spin systems, where a finite temperature is taken into account. First, we will focus on the underlying model and the two basic methods, Langevin dynamics [2] and Monte Carlo simulation [3]. Then we study the magnetization reversal in Co nanoparticles. Starting with the deterministic spin dynamics on short time scales which plays a crucial role in high-speed data storage [4 6], we go on to the probabilistic long-time behavior where a thermally assisted reversal can occur even for magnetic fields below the coercive field [7-11].

\section{Classical Spin Model}

The micromagnetic properties of a nanoparticle can be described using a model of classical magnetic moments which are localized on a given lattice. Such a spin model can be motivated following different lines: on the one hand it is the classical limit of a quantum mechanical, localized spin model — the Heisenberg model [12]. On the other hand, a classical spin model can also be interpreted as the discretized version of a micromagnetic continuum model [13], where the charge distribution for a single cell of the discretized lattice is approximated by a point dipole [12, 14].

The interpretation as an atomic model restricts the use of computer simulations to the investigation of rather small systems of only a few million atoms - corresponding to particle sizes of only a few nanometers. On the other hand, within a continuum model, the space might be discretized on a much larger length scale, as compared to an atomic distance. However, in continuum theory usually a constant absolute value of the magnetization vector is assumed, an assumption which fails for higher temperatures since the space averaged magnetization breaks down when approaching the critical temperature. Hence, one expects correct thermal properties only in the limit of small cell sizes of the order of atomic distances.

In the following, let us consider a classical three dimensional Heisenberg Hamil- 
tonian for localized spins,

$$
\begin{aligned}
\mathcal{H}= & -J \sum_{\langle i j\rangle} \mathbf{S}_{i} \cdot \mathbf{S}_{j}-\mu_{s} \mathbf{B} \cdot \sum_{i} \mathbf{S}_{i}-d_{z} \sum_{i}\left(S_{i}^{z}\right)^{2} \\
& -w \sum_{i<j} \frac{3\left(\mathbf{S}_{i} \cdot \mathbf{e}_{i j}\right)\left(\mathbf{e}_{i j} \cdot \mathbf{S}_{j}\right)-\mathbf{S}_{i} \cdot \mathbf{S}_{j}}{r_{i j}^{3}}
\end{aligned}
$$

where the $\mathbf{S}_{i}=\boldsymbol{\mu}_{i} / \mu_{s}$ are three dimensional magnetic moments of unit length representing atomic magnetic moments. The first sum is the ferromagnetic exchange of the moments with the coupling constant $J$. The second sum is the coupling of the magnetic moments to an external magnetic field $B$, the third sum represents a uniaxial anisotropy, here, for $d_{z}>0$ favoring the $z$ axis as easy axis of the system, and the last sum is the dipolar interaction where $w=\mu_{0} \mu_{s}^{2} /\left(4 \pi a^{3}\right)$ describes the strength of the dipole-dipole interaction. The $\mathbf{e}_{i j}$ are unit vectors pointing from lattice site $i$ to $j$ and $r_{i j}$ is the distance between these lattice sites in units of $a$. The dipole-dipole interaction can be computed efficiently with the help of fast Fourier transformation methods [15, 16]. One should however note that in a Monte Carlo simulation with a single-spin flip algorithm the FFT method is an approximation the implementation of which was described in details before [11.

The transformation of the above introduced atomic parameters to the material parameters which are usually used in a continuum model is given by $J=2 a A_{x}$ where $A_{x}$ is the exchange energy, $d_{z}=K a^{3}$ where $K$ is the anisotropy energy density, and $\mu_{s}=M_{s} a^{3}$ where $M_{s}$ is the spontaneous magnetization.

\section{Landau-Lifshitz Equation and Langevin Dynamics}

In the short time limit spin precession is important which can be taken care of by studying the corresponding equation of motion. The basic numerical approach which includes thermal activation, is the direct numerical integration of the Langevin equation of the problem. In order to obtain thermal averages one has to calculate many of these trajectories starting with the same initial conditions taking an average over these trajectories for the quantities of interest. This method is referred to as the Langevin dynamics formalism [2].

The underlying equation of motion for a magnetic system is the LandauLifshitz-Gilbert (LLG) equation,

$$
\frac{\partial \mathbf{S}_{i}}{\partial t}=-\frac{\gamma}{\left(1+\alpha^{2}\right) \mu_{s}} \mathbf{S}_{i} \times\left[\mathbf{H}_{i}(t)+\alpha\left(\mathbf{S}_{i} \times \mathbf{H}_{i}(t)\right)\right]
$$

with the gyromagnetic ratio $\gamma=1.76 \times 10^{11}(\mathrm{~T} s)^{-1}$, the dimensionless damping constant $\alpha$, and the internal field $\mathbf{H}_{i}(t)=\boldsymbol{\zeta}_{i}(t)-\partial \mathcal{H} / \partial \mathbf{S}_{i}$. Langevin dynamics is 
introduced here in form of the noise $\boldsymbol{\zeta}_{i}(t)$ which represents thermal fluctuations, with $\left\langle\boldsymbol{\zeta}_{i}(t)\right\rangle=0$ and $\left\langle\zeta_{i}^{\eta}(t) \zeta_{j}^{\theta}\left(t^{\prime}\right)\right\rangle=2 \delta_{i j} \delta_{\eta \theta} \delta\left(t-t^{\prime}\right) \alpha k_{\mathrm{B}} T \mu_{s} / \gamma$ where $i, j$ denote once again lattice sites and $\eta, \theta$ Cartesian components.

The LLG equation with Langevin dynamics is a stochastic differential equation with multiplicative noise. For this kind of differential equation a problem arises which is called the Itô-Stratonovich dilemma [17]. As a consequence, different time discretization schemes may converge to different results with decreasing time step. As was pointed out in 18 the multiplicative noise in the Langevin equation above has to be treated by means of the Stratonovich interpretation. An appropriate discretization scheme leading to a Stratonovich interpretation is the Heun method [1, 17, 18] which is used in the following.

\section{Monte Carlo Methods}

In the long time limit only spin relaxation and thermal fluctuations are relevant which can be studied very conveniently using Monte Carlo methods with quantified time step [3]. Within a Monte Carlo approach [19] trajectories in phase space are calculated following a master equation [20] for the time development of the probability distribution $P_{s}(t)$ in phase space,

$$
\frac{\mathrm{d} P_{s}}{\mathrm{~d} t}=\sum_{s^{\prime}}\left(P_{s^{\prime}} w_{s^{\prime} \rightarrow s}-P_{s} w_{s \rightarrow s^{\prime}}\right) .
$$

Here, $s$ and $s^{\prime}$ denote different states of the system and $w_{s^{\prime} \rightarrow s}$ is the transition rate for a change from a state $s^{\prime}$ to a state $s$. These rates have to fulfill the condition [20]

$$
\frac{w_{s \rightarrow s^{\prime}}}{w_{s^{\prime} \rightarrow s}}=\exp \left[\frac{E(S)-E\left(S^{\prime}\right)}{k_{\mathrm{B}} T}\right]
$$

The master equation describes exclusively the coupling of the system to the heat bath [20]. Hence, only the irreversible part of the dynamics of the system is considered including only the relaxation and the fluctuations. A Monte Carlo simulation does not include the energy conserving part of the equation of motion. Hence, no precession of magnetic moments will be found.

Monte Carlo approaches in general have no physical time associated with each step of the algorithm. However, recently a time quantified Monte Carlo method was proposed in [3] and later successfully applied to different model systems [10, 11, 21]. Here, the interpretation of a Monte Carlo step as a realistic time interval $\Delta t$ was achieved by a comparison of one step of the Monte Carlo process with a time interval of the LLG equation in the high damping limit. We will use this algorithm 
in the following. The trial step of this algorithm is a random movement of the magnetic moment within a cone with a given size $r$ with

$$
r^{2}=\frac{20 k_{B} T \alpha \gamma}{\left(1+\alpha^{2}\right) \mu_{s}} \Delta t
$$

In order to achieve this efficiently one constructs a random vector with constant probability distribution within a sphere of radius $r$. This random vector is added to the initial moment and subsequently the resulting vector is normalized [3].

Using this algorithm one Monte Carlo step represents a given time interval $\Delta t$ of the LLG equation in the high damping limit as long as $\Delta t$ is chosen appropriately (for details see [回]).

\section{$5 \quad$ Precessional Reversal in Co Nanoparticles}

In the following we consider Co nanoparticles, where the material parameters are $A_{x}=1.3 \cdot 10^{-11} \mathrm{~J} / \mathrm{m}, K=6.8 \cdot 10^{5} \mathrm{~J} / \mathrm{m}^{2}$ and $M_{s}=1.43 \cdot 10^{6} \mathrm{~A} / \mathrm{m}$. For simplicity we simulate a simple cubic lattice with atomic distance $a=0.25 \mathrm{~nm}$. Our simulation starts with a spin configuration where all magnetic moments point into the $z$ direction, aligned with the easy axis, and with the $z$ component of the external magnetic field antiparallel to the magnetization so that the system is in an unstable, or at least metastable state.

In sufficiently small particles the magnetic moments rotate coherently during the magnetization reversal. A quantitative description of coherent rotation in ellipsoidal single domain particles was developed by Stoner and Wohlfarth [22]. Depending on the angle between the applied field $\mathbf{B}$ and the $z$ (easy) axis of the system, the coercive field $B_{c}$ varies following the so-called Stoner-Wohlfarth asteroid [22]. Under an angle of $45^{\circ}$ it is $B_{c}=d_{\text {eff }} V / \mu_{\mathrm{s}}$ where $V$ is the volume of the particle and $d_{\text {eff }}$ is an effective anisotropy constant. In our case it is $B_{c} \approx 0.7$ T.

First, we are interested in fast switching processes where the applied field is higher than the coercive field and the reversal is dominated by spin precession. We simulate the system with Langevin dynamics as described before. Figure 1 shows the time dependence of the magnetization of our Co particle in the low damping limit $(\alpha=0.1)$ for a very small ellipsoidal shaped particle of length $L=4 \mathrm{~nm}$ and diameter $L=2 \mathrm{~nm}$.

The $z$ and the $x$ components of the magnetization are shown as well as its absolute value. The magnetic field $\mathbf{B}$ is set under an angle of $45^{\circ}$ to the $z$ axis within the $y z$ plane so that the response of the system to the external field sets in directly. The wavering magnetization of the system clearly follows from the precession of the spins. 


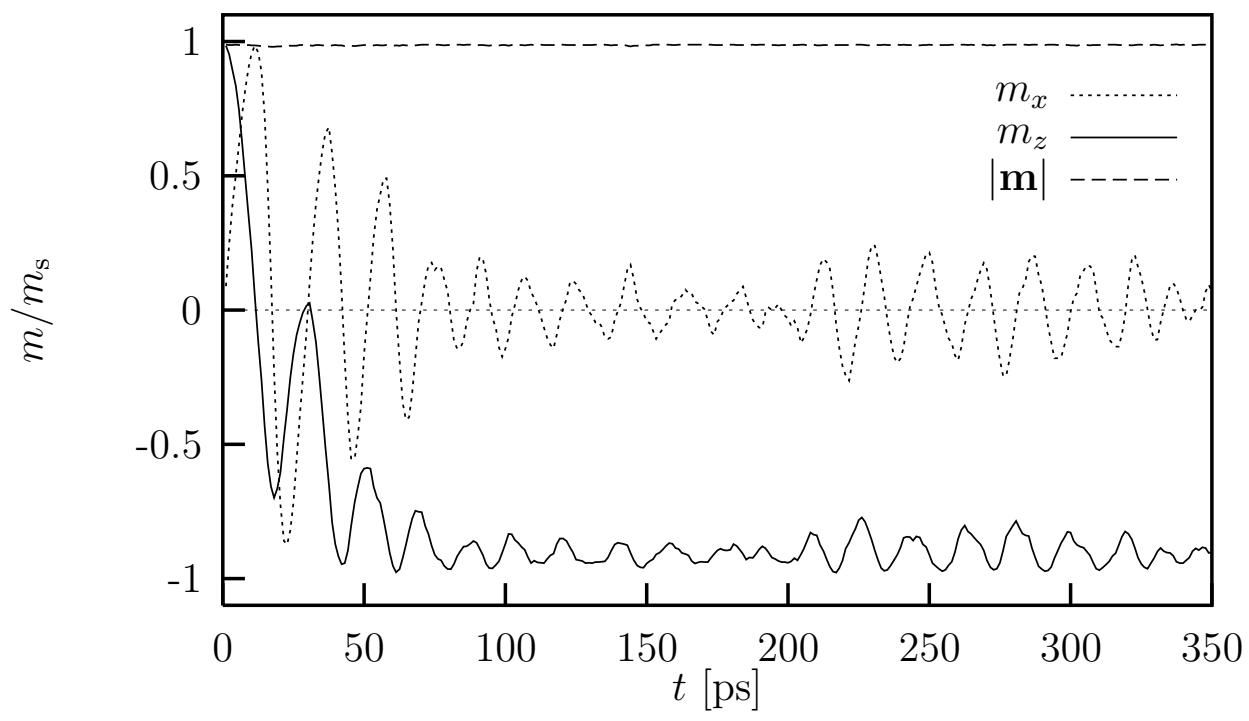

Figure 1: Reduced magnetization vs. time for a Co nanoparticle. The data are from Langevin dynamics simulations. $B=1.13 \mathrm{~T}$ and $T=16 \mathrm{~K}$.

Note, that the precession time of our system is not simply given by the precession time of a single spin in an external field $\left(\tau_{p}=2 \pi\left(1+\alpha^{2}\right) / \gamma B \approx 32 \mathrm{ps}\right.$ in our case). Instead, the whole internal field is relevant for the spin precession, also the contributions from the dipolar field, the exchange and the anisotropy. This internal field is not constant in time and in general it is non-homogenous within the system. However, in the very small particle which we consider here, the internal field is sufficiently homogenous so that the magnetization moves coherently. Hence, the absolute value of the magnetization remains constant in time as is shown in the figure. Note also, that even after the new stable state is reached the magnetization still keeps on oscillating around its equilibrium value, driven by thermal fluctuations.

\section{Thermally Activated Reversal}

Let us now turn to the case $B<B_{c}$, where the reversal process can only occur when it is thermally activated. Since we are now interested in the long time and high damping limit $(\alpha=4)$ where the behavior of the particle is governed by thermal fluctuations we can use Monte Carlo methods. Figure 2 shows the typical time dependence of the magnetization of the same Co particle as before. The field $\mathbf{B}$ is set antiparallel to the initial state here, so that the zero-temperature coercive 


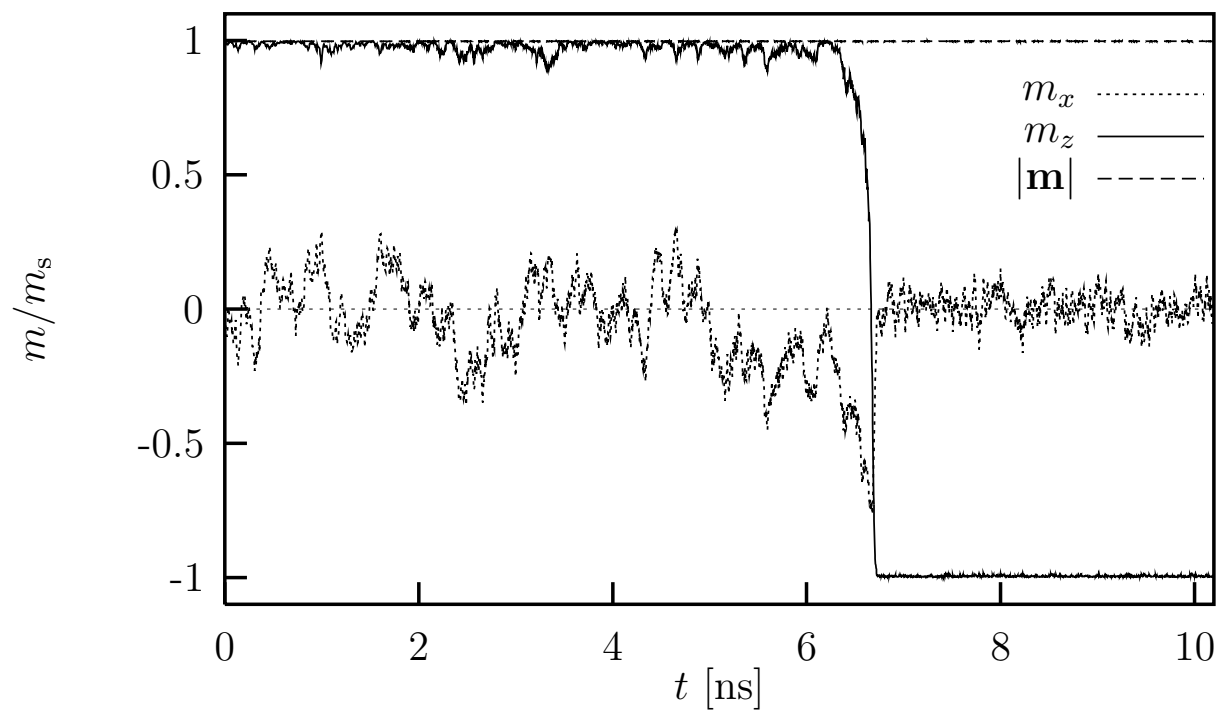

Figure 2: Reduced magnetization vs. time for the same particle as in Fig. 1. The data are from Monte Carlo simulations. $B=1.1 \mathrm{~T}$ and $T=5 \mathrm{~K}$.

field is given by $B_{c}=2 d_{\mathrm{eff}} V / \mu_{\mathrm{s}}$ which in our case is $B_{c} \approx 1.4 \mathrm{~T}$.

As one can see, the $z$ component of the magnetization remains nearly constant for a time period which is rather long as compared to the previous simulation. Then, suddenly, the magnetization drops and its sign changes. As before the absolute value of the magnetization is constant in time and the reversal mechanism is mainly a coherent rotation. The value of the switching time is approximately $6.6 \mathrm{~ns}$ in our simulation. However, this thermally activated switching is not a deterministic process as it was the short time dynamics studied before, where the switching followed mainly from the deterministic part of the equation of motion. Instead, the thermal activation process here is a probabilistic event. The probability distribution $P\left(t_{s}\right)$ for switching events taking place after a time $t_{s}$ follows an exponential law [7],

$$
P\left(t_{s}\right) \sim \exp \left(-t_{\mathrm{s}} / \tau\right)
$$

in the limit of large time-scales. Here, $\tau$ is a characteristic time scale,

$$
\tau=\tau_{0} \exp \left(\Delta E / k_{\mathrm{B}} T\right),
$$

where $\tau_{0}$ is a prefactor and $\Delta E$ an energy barrier which both are related to a certain reversal mechanism (see e. g. [7, 8, 23] for analytically determined prefactors and energy barriers in different systems and [1, 3, 10, 11] for numerical work on this subject). In general the prefactor may depend on the system parameters, the temperature, the applied magnetic field and the damping constant. 


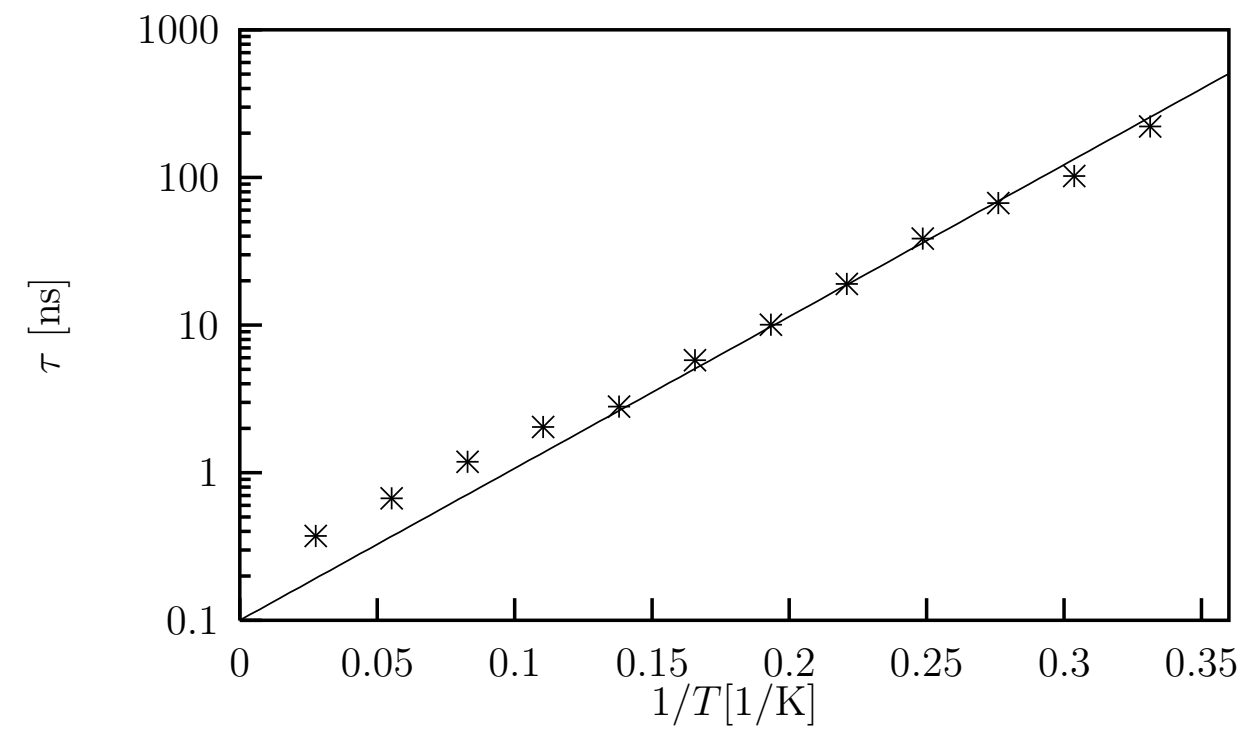

Figure 3: Characteristic time $\tau$ vs. $1 / T$ for the same Co particle as in the figures before. The slope of the solid line represents the energy barrier $\Delta E . B=1.1 \mathrm{~T}$.

For the case of a Stoner-Wohlfarth particle with the applied field parallel to the easy axis the energy barrier has the form

$$
\Delta E=d_{\mathrm{eff}} V\left(1-\frac{B}{B_{c}}\right)^{2} .
$$

This energy barrier as well as the prefactor have been calculated by Brown [7] under the assumption that all magnetic moments are parallel, so that the system behaves like one single magnetic moment.

For a further analysis we extract the energy barrier which governs the reversal process from our numerical data. Figure 3 shows the temperature dependence of the characteristic time, i. e. the mean switching time, obtained from our simulations. The slope of the solid line corresponds to the theoretical value of the energy barrier for a reversal by coherent rotation obtained from Eq. 8. Obviously, it is in very good agreement with our numerical data for low enough temperatures.

\section{Acknowledgments}

This work was supported by the Deutsche Forschungsgemeinschaft (SFB 491 and project NO290/1) and by the European Union (COST action P3, working group $4)$. 


\section{References}

[1] U. Nowak, in Annual Reviews of Computational Physics IX, edited by D. Stauffer (World Scientific, Singapore, 2000), p. 105.

[2] A. Lyberatos and R. W. Chantrell, J. Appl. Phys. 73, 6501 (1993).

[3] U. Nowak, R. W. Chantrell, and E. C. Kennedy, Phys. Rev. Lett. 84, 163 (2000).

[4] C. H. Back, D. Weller, J. Heodmann, D. Mauri, D. Guarisco, E. L. Garwin, and H. C. Siegmann, Phys. Rev. Lett. 81, 3251 (1998).

[5] M. Bauer, J. Fassbender, and B. Hillebrands, J. Appl. Phys. 87, 6274 (2000).

[6] T. Leineweber and H. Kronmüller, Physica B 275, 5 (2000).

[7] W. F. Brown, Phys. Rev. 130, 1677 (1963).

[8] H. B. Braun, Phys. Rev. Lett. 71, 3557 (1993).

[9] W. Wernsdorfer, E. B. Orozco, K. Hasselbach, A. Benoit, B. Barbara, N. Demoncy, A. Loiseau, H. Pascard, and D. Mailly, Phys. Rev. Lett. 78, 1791 (1997).

[10] D. Hinzke and U. Nowak, Phys. Rev. B 61, 6734 (2000).

[11] D. Hinzke and U. Nowak, J. Magn. Magn. Mat. 221, 365 (2000).

[12] A. Aharoni, Introduction to the Theory of Ferromagnetism (Oxford University Press, Oxford, 1996).

[13] T. Schrefl, J. Fidler, R. W. Chantrell, and M. Wongsam, Encyclopedia of Materials: Science and Technology (2001), in press.

[14] A. Hubert and R. Schäfer, Magnetic Domains (Springer-Verlag, Berlin, 1998).

[15] S. W. Yuan and H. N. Bertram, IEEE Trans. Mag. 28, 2031 (1992).

[16] D. V. Berkov, K. R. Ramstöck, and A. Hubert, Phys. stat. sol. (a) 137, 207 (1993).

[17] A. Greiner, W. Strittmatter, and J. Honerkamp, J. Stat. Phys. 51, 95 (1988).

[18] J. L. García-Palacios and F. J. Lázaro, Phys. Rev. B 58, 14937 (1998). 
[19] K. Binder and D. W. Heermann, in Monte Carlo Simulation in Statistical Physics, edited by P. Fulde (Springer-Verlag, Berlin, 1997).

[20] F. Reif, Fundamentals of statistical and thermal physics (McGraw-Hill Book Company, New York, 1965).

[21] R. Smirnov-Rueda, O. Chubykalo, U. Nowak, R. W. Chantrell, and J. M. Gonzáles, J. Appl. Phys. 87, 4798 (2000).

[22] E. C. Stoner and E. P. Wohlfarth, Philos. Trans. R. Soc. London Ser. A 240, 599 (1949).

[23] W. T. Coffey, D. S. F. Crothers, J. L. Dorman, Y. P. Kalmykov, E. C. Kennedy, and W. Wernsdorfer, Phys. Rev. Lett. 80, 5655 (1998). 